\title{
Achieving health gain through clinical guidelines. I: Developing scientifically valid guidelines
}

\author{
Jeremy Grimshaw, Ian Russell
}

There is increasing interest in the role of clinical guidelines in encouraging good practice and thus generating health gain. ${ }^{1}$ However, there is professional ambivalence towards guidelines, stemming mainly from uncertainty about their effectiveness ${ }^{2}$ and how best to introduce them into clinical practice. ${ }^{3}$ Nevertheless, a systematic review of 59 rigorous evaluations of clinical guidelines reported improvements in the process of care in 55 studies and suggested that guidelines can change clinical practice if they are appropriately developed, disseminated, and implemented. ${ }^{4}$ Furthermore, published research has begun to identify effective techniques for introducing clinical guidelines. ${ }^{5-7}$

Following the recent NHS reforms, purchasers are being encouraged to introduce guidelines clarifying the roles of primary and secondary care in patient management. ${ }^{8}$ Purchasers can either collaborate with providers in developing local guidelines or adapt published guidelines for local use. The second option is more attractive since development of a scientifically valid guideline is resource intensive ${ }^{9}$ and the necessary skills are often lacking at a local level. ${ }^{10}{ }^{11}$ However, the quality of published guidelines is variable. In this paper we propose a classification to help purchasers and providers to identify scientifically valid guidelines. Elsewhere we propose a complementary classification to help both parties to ensure that guidelines change medical practice. ${ }^{11 a}$

\section{Guidelines, criteria, and standards}

Quality assurance is beset with problems of terminology; terms used as synonyms include guidelines, algorithms, consensus statements, criteria, protocols, and standards. For example, the term standard is commonly used in medical audit to describe "performance the auditors have set themselves to achieve." 12 However, it has also been used to describe "complex aggregations of criteria built up into a series of statements describing good clinical practice." ${ }^{13}$ Commenting on the terminology of quality assurance, Donabedian noted that "we have used these words in so many different ways that we no longer clearly understand each other when we say them" and suggested that we need to "clarify the existing nomenclature, encrusted and misshapen though it may be." 14 Fortunately, the US Agency for Health Care Policy and Research (AHCPR) has recently agreed distinct definitions for "practice guidelines," "medical review criteria," and "standards of quality."15
PRACTICE GUIDELINES

Practice guidelines are "systematically developed statements to assist practitioner and patient decisions about appropriate health care for specific clinical circumstances." 15 Such guidelines expound appropriate management options for symptom clusters, conditions, or procedures with the principal aim of promoting good performance.

Guidelines are composed of elements describing different aspects of the patient's condition and the care to be given. Eddy argued that the relative importance of these elements should be based on the weight of scientific evidence linking them to the desired clinical outcome. ${ }^{16} \mathrm{He}$ distinguished between three levels of importance: "standards" (in his terminology) define appropriate care and should be followed in all circumstances with no flexibility for the clinician; "guidelines" should be followed in most circumstances but allow some flexibility in some circumstances; and "options" are "so flexible as to provide virtually no guidance at all."16

Although this link between levels of importance and flexibility is helpful, Eddy's terminology is perhaps not as helpful as Irvine and Donaldson's alternative suggestion of mandatory, near mandatory, and optional elements. ${ }^{13}$ Elements which are well founded scientifically and have important implications for patient outcome should be mandatory. In the management of acute myocardial infarction (AMI), for example, early administration of aspirin has been shown conclusively to reduce subsequent mortality. ${ }^{17}$ Thus a guideline for the management of suspected AMI should include a mandatory element requiring the early administration of aspirin to all patients without specific contraindications. In contrast, where there are alternative management strategies but no scientific evidence about relative effectiveness, the element should be considered optional, signalling a need for research to identify appropriate management. In clinical practice there is a strong case for asking patients about their preferences between these "options."

Guidelines vary in the amount of operational detail included. Those developed nationally or regionally will generally reflect broad statements of good clinical practice with little operational detail. They need to be adapted to reflect the local context and available resources. In Scotland the Clinical Resource and Audit Group has proposed that national guidelines are modified to produce local protocols - that is, detailed developments 
of (nationally derived guidelines) for local application."18 The distinction between national and local guidelines based on the degree of operational detail is useful; however, the term protocol may not be acceptable to all.

Guidelines can be deterministic or branching in structure. ${ }^{19}$ Deterministic guidelines comprise a fixed list of elements which should be followed, irrespective of the information available to the doctor. In branching guidelines, however, the recommended course of action at each stage depends critically on the available information. Though deterministic guidelines may be appropriate to define minimum levels of care, they ignore the deductive nature of medical decision making and are therefore less appropriate to guide routine clinical practice. ${ }^{19}$ Branching guidelines are commonly presented either as algorithms (or "flow charts") or as prose statements. The choice between these formats usually depends upon the topic and the target audience. In the North of England study, peer groups of general practitioners showed considerable diversity in the style of their internal guidelines. ${ }^{20}$ In contrast, the Harvard Community Health Plan has established a quality assurance programme based exclusively on algorithms, ${ }^{21}$ building on their successful use as a method of information transfer in educational settings. ${ }^{22}$ However, doctors are often reluctant to use algorithms in everyday practice because of their apparent complexity and lack of flexibility.

MEDICAL REVIEW CRITERIA

Medical review criteria are "systematically developed statements that can be used to assess the appropriateness of specific health care decisions, services, and outcomes."15 Such criteria may either be developed specifically to assess appropriateness of care (see, for example, Park et $a l^{23}$ ) or be derived from clinical guidelines that were introduced for contracting or quality assurance; the latter can be selected either during the development of the guidelines or later by those monitoring contracts or coordinating quality assurance. These criteria may relate to all elements of care within a guideline or to a representative sample of elements. In general, it is desirable to define a set of criteria that cover the important aspects of the guidelines. We propose that such criteria should fulfil the requirements listed in the box and be based on mandatory or, at worst, near mandatory elements. In the management of suspected AMI, for example, a useful criterion for assessing performance after the introduction of guidelines is the percentage of patients without contraindications receiving aspirin within two hours of contacting a health professional.

STANDARDS OF QUALITY

Standards of quality are "authoritative statements of (a) minimum levels of acceptable performance or results, (b) excellent levels of performance or results, or (c) the range of acceptable performance or results." Irvine and Donaldson proposed alternative terms for the United Kingdom: ideal standards reflect "the best attainable under the very best conditions, where money and resources are unlimited"; optimal standards are "the best which conscientious practitioners can achieve under (normal) working conditions with the resources that are available to them"; and minimal standards are "those below which care should not be allowed to fall without causing the possibility of harm to patients." 13

Standards relate to medical review criteria rather than to guidelines. Once criteria have been selected, a desired standard of performance can be defined. The standard chosen will depend on both the relative importance of the element from which the criterion is derived and the desired "level" of performance. The ideal standard may be interpreted as the estimated level beyond which iatrogenesis occurs, and the minimal standard as the estimated level below which avoidable mortality or major morbidity occurs. We believe, however, that it is the optimal standard at which both purchasers and providers should aim; this is best interpreted as the level at which the resources devoted to fulfilling the guideline give value (to patients) for money equal to that if they were devoted to any other NHS activity.

In the management of suspected AMI, for example, the standard for a mandatory element (for example, the percentage of patients without contraindications receiving aspirin within two hours of contacting a health professional) could be as high as $99 \%$ for ideal performance, as low as $75 \%$ for minimal performance, and between $85 \%$ and $95 \%$ for optimal performance. The use of a confidence-like interval for the optimal standard acknowledges the difficulty of

\section{Requirements for medical review} criteria

1 Each criterion should be easy to define ${ }^{\star}$

2 Each criterion should relate to morbidity that is amenable to improvement by medical care $^{\star}$

3 There should be a sound scientific basis for discriminating between good and less than good performance as judged by each criterion ${ }^{\star}$

4 The effects of non-medical factors on this performance should be adequately understood ${ }^{\star}$

5 The criterion should yield data on enough patients for valid statistical analysis*

6 Together these criteria should span the range of morbidity covered by the guidelinet

7 Together these criteria should span the range of skills required by the guidelinet

8 Together these criteria should span the range of resources specified by the guideline $\dagger$

$\star$ Derived from Kessner $e t a l^{24}$ + Derived from Irvine ${ }^{25}$ 
Table 1 Desirable attributes of clinical guidelines*

\begin{tabular}{|c|c|}
\hline Attribute & Explanation \\
\hline Validity & $\begin{array}{l}\text { Guidelines are valid if, when followed, they lead to the health } \\
\text { gains and costs predicted for them }\end{array}$ \\
\hline Reproducibility & $\begin{array}{l}\text { Guidelines are reproducible if, given the same evidence and } \\
\text { methods of guideline development, another guideline group } \\
\text { produces essentially the same recommendations }\end{array}$ \\
\hline Reliability & $\begin{array}{l}\text { Guidelines are reliable if, given the same clinical } \\
\text { circumstances, another health professional interprets and } \\
\text { applies them in essentially the same way }\end{array}$ \\
\hline Representative development & $\begin{array}{l}\text { Guidelines should be developed by a process that entails } \\
\text { participation by key affected groups }\end{array}$ \\
\hline Clinical applicability & $\begin{array}{l}\text { Guidelines should apply to patient populations defined in } \\
\text { accordance with scientific evidence or best clinical judgement }\end{array}$ \\
\hline Clinical flexibility & $\begin{array}{l}\text { Guidelines should identify exceptions to their } \\
\text { recommendations and indicate how patient preferences are to } \\
\text { be incorporated in decision making }\end{array}$ \\
\hline Clarity & $\begin{array}{l}\text { Guidelines must use unambiguous language, precise } \\
\text { definitions, and user friendly formats }\end{array}$ \\
\hline Meticulous documentation & $\begin{array}{l}\text { Guidelines must record participants involved, assumptions } \\
\text { made, and evidence and methods used }\end{array}$ \\
\hline Scheduled review & $\begin{array}{l}\text { Guidelines must state when and how they are to be reviewed } \\
\text { (under two separate circumstances - the identification or not } \\
\text { of new scientific evidence or professional consensus) }\end{array}$ \\
\hline
\end{tabular}

estimating the value for money of a range of alternative uses of NHS resources given the current state of the knowledge base about health service effectiveness and efficiency. It is this knowledge base that the NHS research and development programme is designed to enhance.

\section{Factors influencing the validity of guidelines}

To maximise the potential health gain from guidelines it is important to ensure that they are rigorously developed and thus consistent with the available scientific evidence or, in the absence of such evidence, best clinical judgement. If those developing guidelines fail to overcome the many potential biases inherent in that development, the resulting guidelines may recommend ineffective or even dangerous clinical practice. In this paper, therefore, we propose a classification which links validity to the way in which guidelines are developed. However, this classification does not cover dissemination and implementation strategies as these are not intrinsic properties of a guideline despite their importance in determining whether guidelines achieve health gain. ${ }^{11 a}$

Table 1 summarises nine desirable attributes for clinical guidelines proposed by the AHCPR ${ }^{15}$ Although only the first of these is labelled "validity," our systematic review of clinical guidelines ${ }^{4}$ suggests that the next three attributes - reproducibility, reliability, and representative development - also affect whether or not guidelines lead to health gains when they are followed. (In other words, as others have noted in other contexts, validity, reproducibility, reliability, and representativeness are not independent attributes.)

While those developing guidelines should aim at achieving all nine attributes and focusing especially on the first four, we are conscious that these four are difficult to bring into operation, especially for purchasers and providers. Table 2 therefore identifies four practical questions that, published work suggests, have a direct, though variable, effect on validity: how evidence was identified and synthesised; how many users of guidelines and how many key disciplines were included in the guideline group; and how the guidelines were developed.

Even with the help of this table, it is difficult for potential users to assess published guidelines; contrary to the penultimate attribute of table 1 (meticulous documentation), few guidelines include details of their development. Of the 59 rigorous evaluations of guidelines in our review, ${ }^{4}$ none gave sufficient information to enable us to judge with confidence the rigour of their development. Fortunately, Hayward and colleagues have recently proposed structured abstracts for guidelines, which would encourage developers to publish details including methods used to gather, select, and synthesise evidence; methods used to assign values (relative importance) to the potential outcomes of alternative practice options; and validation (for example, results of any external review of the resulting guidelines, comparison with guidelines developed by other groups, and clinical testing of guidelines in practice). ${ }^{26}$

METHODS OF IDENTIFYING AND SYNTHESISING EVIDENCE

Methods of identifying and synthesising evidence include expert opinion, unsystematic literature review, ungraded systematic review, graded systematic review, and formal metaanalysis (table 2). In the United Kingdom many guidelines are currently developed by expert groups without formal literature reviews. This approach relies heavily upon the group's knowledge of published work and their clinical experiences in non-evaluative

Table 2 Factors influencing the validity of guidelines

\begin{tabular}{|c|c|c|c|c|}
\hline \multirow{2}{*}{$\begin{array}{l}\text { Likelihood of } \\
\text { scientific } \\
\text { validity }\end{array}$} & \multirow[t]{2}{*}{ Method of synthesising evidence } & \multicolumn{2}{|c|}{ Composition of guideline group } & \multirow{2}{*}{$\begin{array}{l}\text { Method of developing } \\
\text { guidelines }\end{array}$} \\
\hline & & $\begin{array}{l}\text { Proportion of users } \\
\text { of guidelines }\end{array}$ & $\begin{array}{l}\text { No of key } \\
\text { disciplines } \\
\text { represented }\end{array}$ & \\
\hline \multirow[t]{2}{*}{ High } & Formal meta-analysis & $\begin{array}{l}\text { Low ("national external" } \\
\text { guideline group) }\end{array}$ & All & $\begin{array}{l}\text { Evidence linked } \\
\text { guideline development }\end{array}$ \\
\hline & Graded systematic review & $\begin{array}{l}\text { Low ("local external" } \\
\text { guideline group) }\end{array}$ & & \\
\hline \multirow[t]{2}{*}{ Medium } & Ungraded systematic review & $\begin{array}{l}\text { Medium ("intermediate" } \\
\text { guideline group) }\end{array}$ & Some & $\begin{array}{l}\text { Formal consensus } \\
\text { development }\end{array}$ \\
\hline & Unsystematic review & & & \\
\hline Low & Expert opinion & $\begin{array}{l}\text { High ("internal" guideline } \\
\text { group) }\end{array}$ & One & $\begin{array}{l}\text { Informal consensus } \\
\text { development }\end{array}$ \\
\hline
\end{tabular}


conditions. However, clinicians' knowledge of published work is often incomplete, through poor presentation of research findings, ${ }^{27}$ lack of time to search for information, and dispersion or scatter of relevant literature across a large number of journals. ${ }^{28}$ Furthermore, clinicians have difficulty in interpreting published evidence. ${ }^{27}$ The value of clinicians' experiences have also been questioned. Sackett identified four reasons why clinical experience may be biased and overestimate effectiveness: correlation between patient compliance and response to treatment; regression to the mean; the placebo effect; and the desire of both patients and clinicians for a successful response. ${ }^{29}$ Thus guidelines developed without a literature review may be biased towards reinforcing current practice rather than promoting evidence-based practice.

Traditional unsystematic literature reviews (without explicit research strategies, explicit inclusion criteria, or formal methods of synthesising evidence) overcome some of these problems by expanding the evidence base. However, important studies may be missed if the implicit search strategies are inappropriate or incomplete. Furthermore, the lack of explicit inclusion criteria can overwhelm the reviewer with evidence of variable quality, and the lack of formal methods of synthesis create difficulties in interpreting the evidence. Thus guidelines developed after an unsystematic literature review may suffer from selection bias while providing false reassurance.

Systematic reviews overcome these problems by defining search strategies and inclusion criteria - for example, for research designs. Ungraded systematic reviews use explicit standards to judge the scientific validity and clinicial applicability of evidence and thus draw conclusions only from studies that are judged methodologically sound. For example, Haynes and colleagues reviewed the literature evaluating the effectiveness of continuing medical education. ${ }^{30}$ Before starting they defined an explicit search strategy and inclusion criteria specifying a minimum standard of methodological rigour. They identified 248 potential studies published between 1970 and 1983, of which only seven studies fulfilled their inclusion criteria. This allowed them to ensure that their conclusions were based on the best scientific evidence.

Ungraded systematic reviews typically review only randomised trials, but this approach may result in important evidence from other research designs being ignored. Sackett therefore described a method of grading evidence in which studies are ranked by design - for example, level I indicates a large randomised trial with low risk of error and level $\mathrm{V}$ a series of cases with no control data. ${ }^{29}$ This allows those developing guidelines to grade recommendations according to the strength of evidence - for example, a grade A recommendation is supported by at least one level I study.

When contradictory results are reported by trials addressing similar issues or several trials need to be combined formal meta-analysis is recommended. ${ }^{31}$ This combines the data or results of multiple trials and summarises all the reviewed evidence by a single statistic, typically a pooled relative risk of mortality (see for example, Yusef et $a l^{32}$ ). Technologies successfully evaluated in this way include $\beta$ blockade after myocardial infarction ${ }^{32}$ and prophylactic antibiotics in caesarean section ${ }^{33}$; in contrast, technologies on which metaanalysis has thrown doubt include spinal manipulation for back and neck pain $^{34}$ and lipid lowering drugs in low risk patients. ${ }^{35}$ Most meta-analyses consider only randomised trials and are thus open to the same criticism as ungraded systematic reviews. However, a generalisation of Sackett's proposal ${ }^{29}$ should in due course enable reviewers to derive pooled estimates from graded systematic reviews.

\section{COMPOSITION OF GUIDELINE DEVELOPMENT}

GROUP

Both the validity and acceptability of guidelines depend on whether they are developed by an internal group comprising the clinicians who are going to use them, an intermediate group including representatives of those clinicians, or an external group excluding those clinicians. External guidelines may be produced either locally or nationally. Though guidelines developed internally are more likely to change medical practice, ${ }^{11 a}$ they are less likely to be scientifically valid through lack of technical skills in guideline development, ${ }^{10} 11$ clinical expertise, and resources. ${ }^{9}$

The balance of disciplines within a guideline development group also has considerable influence on the recommendations. Leape and colleagues observed differences in recommendations for carotid endarterectomy between a surgical panel and a "balanced panel" comprising surgeons, neurologists, and other specialists: the surgical panel found more indications for surgery than the balanced panel. ${ }^{36}$ Scott and Black observed similar differences between surgical and mixed panels working on cholecystectomy. ${ }^{37}$ Lomas identified three reasons why guideline development groups should include representatives of all "stakeholders": firstly, the limited information available for guideline development needs to be supplemented by the interpretations of these stakeholders; secondly, legitimate conflicts over values need to be resolved; and thirdly, the successful introduction of a guideline requires that all key disciplines contribute to its development to ensure "ownership" and support. ${ }^{38}$

\section{METHODS OF DEVELOPING GUIDELINES}

There are three main methods of developing guidelines: peer groups, Delphi techniques, and consensus conferences. ${ }^{39} 40$ However, all three suffer from biases and there is little evidence on their relative merits. ${ }^{11}$ Thus it is inappropriate to use the primary method of development as a classification factor.

Woolf identified four secondary methods of development distinguished by the nature of decision making: informal consensus development, formal consensus development, 
evidence linked guideline development, and explicit guideline development. ${ }^{41}$ In informal consensus development the guideline development group has poorly defined, often implicit, criteria for decision making; Eddy described this approach as global subjective judgement. ${ }^{42}$ This approach has been commonly used to develop guidelines in the United Kingdom. ${ }^{43}$ It is free of complex analytical procedures and therefore relatively easy and fast. However, the guidelines are often of poor quality because the lack of explicit consensus method can lead to the group being dominated by forceful personalities or interests. Furthermore, guidelines produced by this method are often poorly documented, with little methodological information to reassure the user about scientific validity.

Formal consensus development methods (used by many consensus development conferences and Delphi groups) provides "greater structure to the analytical process" but fails to provide "an explicit linkage between recommendations and quality of evidence." 41 Evidence-linked guideline development requires the explicit linkage of recommendations to the quality of the supporting evidence (see, for example, Woolf $e t a^{44}$ ), and thus enhances the scientific validity of the guideline. In the absence of rigorous evidence it is even possible to include expert opinion within this approach if the resulting recommendations are clearly documented. Explicit guideline development (perhaps better described as "evaluative" guideline development) evaluates the benefits, risks, and costs of potential interventions by estimating the probability and value of each potential outcome and thus develops a "balance sheet" to facilitate patients' decisions about alternative treatments. ${ }^{45}$ While this approach is attractive in theory, its use in practice is so limited that it is not yet appropriate to include it with other methods of synthesising evidence in table 2 .

\section{Conclusion}

Within the United Kingdom the development and use of clinical guidelines is relatively new. As a result, few guidelines have been developed rigorously. If the introduction of guidelines into contracts is to generate health gain, however, purchasers and providers need to identify rigorously developed guidelines. In this paper we have proposed a classification of factors influencing the scientific validity of guidelines designed to inform choice about which guidelines to be integrated into contracts. Greater validity is likely to follow from the use of systematic literature reviews, independent (external) guideline development groups including representatives of all key disciplines, and the explicit links between recommendations and scientific evidence. Those developing future guidelines should also adopt these strategies whenever possible.

We thank the Chief Scientist Office of the Home and Health Department of the Scottish Office for funding the Health Services Research Unit; however the opinions expressed in this paper are those of the authors, not of the funding body.
Delamothe T. Wanted: guidelines that doctors will follow. $B M F$ 1993;307:218.

Guidelines for doctors in the new world Lancet 1992; 339:1197-8

3 Haines A, Feder G. Guidance on guidelines: writing them is easier than making them work. $B M \mathcal{F} 1992$ 305:785-6.

4 Grimshaw JM, Russell IT. Do clinical guidelines change medical practice? A systematic review of rigorous evaluations. Lancet (in press).

5 Lomas J, Haynes RB. A taxonomy and critical review of tested strategies for the application of clinical practice recommendations: from "official" to "individual" clinical policy. Am f Prev Med 1987;4:77-94.

6 Grol R. Implementing guidelines in general practice care. Quality in Health Care 1992;1:184-91.

7 Mittman BS, Tonesk X, Jacobson PD. Implementing clinical practice guidelines: social influence strategies and clinical practice guidelines: social influence strategies an
practitioner behaviour change. $Q R B$ 1992;18:413-22.

8 Sheldon TA, Borowitz M. Changing the measure of quality in the NHS: from purchasing activity to purchasing protocols. Quality in Health Care 1993;2:149-50.

9 Brook RH. Practice guidelines and practising medicine: are they compatible? $\mathfrak{F} A M A 1989 ; 262: 3027-30$.

10 Grol R. Quality assurance: approaches to standard setting assessment and change. Aten Primaria 1990;7:737-41.

11 Russell IT, Grimshaw JM, Wilson BJ. Epidemiological and statistical issues in quality assurance. In: Beck JS, Bouchier IAD, Russell IT, eds. Quality assurance in medical care. Edinburgh: Royal Society of Edinburgh (in press).

11a Grimshaw JM, Russell IT. Achieving health gain through clinical guidelines. II. Ensuring that guidelines change medical practice. Quality in Health Care (in press).

12 Marinker M. Standards. In: Marinker M, ed. Medical audit and general practice. London: BMF, 1990.

13 Irvine D, Donaldson L. Quality and standards in health care. In: Beck JS, Bouchier IAD, Russell IT, eds. Quality assurance in medical care. Edinburgh: Royal Society of Edinburgh (in press).

14 Donabedian A. Explorations in quality assessment and monitoring. II. The criteria and standards of quality. Ann Arbor, Michigan: Health Administration Press, 1982.

15 Institute of Medicine. Guidelines for clinical practice: from development to use. Washington, DC: National Academic Press, 1992.

16 Eddy DM. Practice policies - what are they? $\mathcal{F} A M A$ 1990;263:877-80.

17 Second International Study of Infarct Survival Collaborative Group. Randomised trial of intravenous streptokinase, oral aspirin, both, or neither among 17187 cases of suspected myocardial infarction: ISIS-2 17187 cases of suspected

18 Clinical Resource and Audit Group. Clinical guidelines: a report by a working group set up by the Clinical Resource and Audit Group. Edinburgh: Scottish Office, 1993.

19 Irvine DH, Russell IT, Hutchinson A, et al. Performance review in general practice: educational development and evaluative research in the Northern region. In: Pendleton $\mathrm{DA}$, Schofield TPC, Marinker ML, eds. In pursuit of quality? London: Royal College of General Practitioners, 1986 .

20 North of England Study of Standards and Performance in General Practice. Overview of the study. Newcastle upon Tyne: Centre for Health Services Research, 1991. (Report 50.)

21 Schoenbaum SC, Gottlieb LK. Algorithm based improvement of clinical quality. BMF 1990;301:1374-6.

22 Margolis CZ, Cook CD, Barak N, Adler A, Geertsma A Clinical algorithms teach pediatric decision making more Clinical algorithms teach pediatric decision making

23 Park RE, Fink A, Brook RH, et al. Physician ratings of appropriate indications for six medical and surgical procedures. Am F Public Health 1986;76:766-72.

24 Kessner DM, Kalk CE, Singer AA. Assessing health quality - the case for tracers. $N$ Engl f Med 1973;288:189-94.

25 Irvine D. Managing for quality in general practice. London King's Fund Centre, 1990. (Medical Audit Series 2.)

26 Hayward RSA, Wilson MC, Tunis SR, Bass EB, Rubin HR, Haynes RB. More informative abstracts of articles describing clinical practice guidelines. Ann Intern Med 1993;118:731-7.

27 Haynes RB, Sackett DL, Tugwell P. Problems in handling of clinical research evidence by medical practitioners. Arch Intern Med 1983;143:1971-5.

28 Haynes RB. Loose connections between peer-reviewed clinical journals and clinical practice. Ann Intern Med clinical journals

29 Sackett DL. Rules of evidence and clinical recommendations on the use of antithrombotic agents.

30 Haynes RB, Roms DA, McKibbon A, Tugwell P. A critical appraisal of the efficacy of continuing medical education. IAMA 1984;251:61-4.

31 Chalmers I. Evaluating the effects of care during pregnancy and childbirth. In: Chalmers I, Enkin M, Keirse MJNC eds. Effective care in pregnancy and childbirth. 1. Pregnancy. Oxford: Oxford University Press, 1989:3-38.

32 Yusef $S$, Peto $R$, Lewis J, Collins $R$, Sleight P. Beta blockade during and after myocardial infarction: an overview of the randomised trials. Prog Cardiovasc Di 1985;27:335-71.

33 Enkin M, Enkin E, Chalmers I, Hemminki E. Prophylactic antibiotics in association with caesarean section. In Chalmers I, Enkin M, Keirse MJNC, eds. Effective care 
in pregnancy and childbirth. 2. Childbirth. Oxford: Oxford University Press, 1989:1246-69.

34 Koes BW, Assendelft WIJ van der Heijden GJMG, Bouter LM, Knipschild PG. Spinal manipulation and mobilisation for back and neck pain: a blinded review.

35 Davey Smith G, Song F, Sheldon TA. Cholesterol lowering and mortality: the importance of considering initial level of risk. $B M \mathcal{F}$ 1993;306:1367-73

36 Leape LL, Park RE, Kahan JP, Brook RH. Group judgements of appropriateness: the effect of pane composition. Qual Assur Health Care 1992;4:151-9.

37 Scott EA, Black N. When does consensus exist in expert panels? F Public Health Med 1991;13:35-9.

38 Lomas J. Making clinical policy explicit: legislative policy making and lessons for developing practice guidelines. Int f Technol Assess Health Care 1993;9:11-25.

39 Audet AM, Greenfield S, Field M. Medical practice guidelines: current activities and future directions. Ann guidelines: current activities a
Intern Med 1990;113:709-14.
40 Fink A, Kosecoff J, Chassin M, Brook RH. Consensus methods: characteristics and guidelines for use. $A m \mathcal{F}$ methods: characteristics and guric
Public Health 1984;74:979-83.

41 Woolf SH. Practice guidelines - a new reality in medicine. II. Methods of developing guidelines. Arch Intern Med 1992;152:946-52.

42 Eddy DM. Practice policies: where do they come from? ЭAMA 1990;263:1265-75.

43 Newton JC, Hutchinson A, Steen IN, Russell IT, Haines EV. Educational potential of medical audit: observations from a study of small groups setting standards. Quality in Health Care 1992;1:256-9.

44 Woolf SH, Battista RN, Anderson GM, Logan AG, Wang E. Assessing the effectiveness of preventive manoeuvres: analytic principles and systematic methods in reviewing evidence and developing clinical practice recommendations. F Clin Epidemiol 1990;43:891-905.

45 Eddy DM. Comparing benefits and harms: the balance sheet. $¥ A M A$ 1990;263:2239-43. 